\title{
A simplified method to determine the first primary drying and wetting curves of water diffusivity of unsaturated soil
}

\author{
A. Sommella, ${ }^{1}$ A. Comegna, ${ }^{2}$ M. Palladino, ${ }^{1}$ A. Coppola ${ }^{3}$ \\ ${ }^{1}$ Division of Water Resources Management, University of Naples "Federico II", Italy; ${ }^{2}$ School of \\ Agricultural Forestry Food and Environmental Sciences (SAFE), University of Basilicata, Potenza, \\ Italy; ${ }^{3}$ Department of European and Mediterranean Cultures-Architecture, Environment, Cultural \\ Heritage (DiCEM), Hydraulics and Hydrology Division, University of Basilicata, Matera, Italy
}

\begin{abstract}
Within the framework of a research project examining the spatial variability of hydraulic characteristics of soil intended for irrigation, some of the more frequently used analytical expressions describing the laws linking diffusivity $D$ to the water content of the soil $\theta$ were verified. By studying the flow field of soil samples tested in the laboratory, under one-dimensional wetting and drying cycles, it has been found that the laws of hydraulic diffusivity of the exponential types can be ascribed to them. Finally, a simplified laboratory method was proposed which, with the aid of nomographs, allows the definition of the law $\mathrm{D}(\theta)$ to be easily arrived at.
\end{abstract}

\section{Introduction}

Soil hydraulic properties such as the soil water retention curve $\mathrm{h}(\theta)$, unsaturated hydraulic conductivity $\mathrm{K}(\theta)$ and diffusivity $\mathrm{D}(\theta)$, are very important in all computational models for predicting water flow. Of the three hydraulic properties, only two are necessary since any of the three can be obtained from the other two which are independent. Direct measurements of these properties is quite expensive and time-consuming.

Methods to measure soil water diffusivity in the laboratory have been extensively explored. Initially Gardner (1956) presented a method for experimentally calculating diffusivity from volumetric measurements of water outflow with time from a soil sample subjected to an abrupt change in soil water pressure. The impedance to flow

Correspondence: A. Comegna, School of Agricultural Forestry Food and Environmental Sciences (SAFE), University of Basilicata, Potenza, Italy. E-mail: alessandro.comegna@unibas.it

Key words: soil hydraulic characterization, soil water diffusivity, laboratory method.

(C) Copyright A. Sommella et al., 2013

Licensee PAGEPress, Italy

Journal of Agricultural Engineering 2013; XLIV(s2):e168

doi:10.4081/jae.2013.s2.e168

This article is distributed under the terms of the Creative Commons Attribution Noncommercial License (by-nc 3.0) which permits any noncommercial use, distribution, and reproduction in any medium, provided the original author(s) and source are credited. offered by the membrane was neglected in his mathematical description. Miller and Elrick (1958) improved this pressure outflow method by including membrane impedance in the solution. Rijtema (1959) presented a procedure for obtaining the membrane and soil contact impedance from the experimental data. The simplified method of Kunze and Kirkham (1962) not only reduced the number of steps required to calculate the diffusivity but eliminated the necessity of measuring membrane impedance. Experimental and theoretical determination of the diffusivity by the above methods requires three basic assumptions: i) soil water content be a linear function of the soil water pressure within the applied pressure increment, ii) that diffusivity be constant throughout the imposed water content range; and iii) that Darcy's law be applicable to unsaturated soil water movement.

Gardner (1962) presented the pressure outflow equation in a slightly different form by assuming that water content does not vary appreciably with sample depth during outflow and calculated the diffusivity and average water content directly from the instantaneous outflow rate. This procedure has been used by Doering (1965), Gupta et al. (1974), Passioura (1976), Valiantzas (1990), and Londra and Valiantzas (2011), and is referred to as the one-step outflow method which can be ascribed to so-called direct laboratory methods. On the other hand, within the indirect approach, parameter estimation methods have been applied together with laboratory outflow experiments to determine hydraulic properties (Kool et al., 1987; Parker et al., 1985; Eching et al., 1994; Ciollaro and Romano, 1995). Parameter estimation techniques enable simultaneous determination of the functions $h(\theta)$, $\mathrm{K}(\theta)$ and $\mathrm{D}(\theta)$ just from outflow experiments. An assumption of particular mathematical forms of the hydraulic properties is however required. The indirect method is certainly more flexible than the direct one, but nonetheless there are some drawbacks concerning the way the problem is posed, instability and lack of solution uniqueness.

The above procedures assume that volumetric water content $\theta$ is uniquely related to the water potential $h$ during the drying and wetting process. However, in unsaturated zones of the flow domain, the potential $h$ is not a unique function of $\theta$ and thus hydraulic characterisation of the medium extended to hysteresis phenomena envisages, at the very least, definition of the two primary functions of $\mathrm{D}(\theta)$ and $K(\theta)$, respectively of wetting and drying.

Given the onerous task of conducting laboratory tests required for complete soil hydraulic characterisation, in this work we examined the possibility of characterising soil samples through exponential laws obtained with more straightforward, rapid testing techniques. Thus we examined the wetting and drying processes in the laboratory, using a vertical experiment, and the results of tests were compared with the theoretical solutions of flow domains supplied by Scott and Hanks (1962) and obtained by approximating the law $D(\theta)$ through an exponential expression suggested by Klute et al. (1965).

Contrary to the conventional one-step outflow method the present method requires only the measurement of the average volumetric soil 
water content. The advantage of the proposed method over existing methods is that it provides a rapid, easy method to determine $D(\theta)$ without requiring the laborious recording and analysis of all outflow volumes over time.

\section{Theory}

For one-dimensional vertical flow the continuity equation takes the form:

$$
\frac{\partial \theta}{\partial \mathrm{t}}=\frac{\partial \mathrm{q}}{\partial \mathrm{z}}
$$

where $\theta$ is volumetric soil water content, q is the soil water flux density, $\mathrm{z}$ is taken positive downwards and $t$ is time. Combined with the generalized Darcy law:

$\mathrm{q}=-\mathrm{K}(\theta) \frac{\partial \mathrm{H}}{\partial \mathrm{z}}$

the soil water transfer equation can be written as:

$$
\frac{\partial \theta}{\partial \mathrm{t}}=\frac{\partial}{\partial \mathrm{z}}\left[\mathrm{K}(\theta)\left(\frac{\partial \mathrm{h}}{\partial \mathrm{z}}-1\right)\right]
$$

where $\mathrm{h}$ is soil water pressure head relative to atmospheric pressure (h $\leq 0$ ), $\mathrm{K}$ is hydraulic conductivity as a function of $\theta$, and $\mathrm{H}$ is hydraulic head defined as:

$$
\mathrm{H}=\mathrm{h}(\theta)-\mathrm{z}
$$

For non-swelling soil (Childs and Collins-George, 1950; Coppola et al., 2012), equation 3 can be expressed as a $\theta$ dependent equation by introducing the concept of soil water diffusivity:

$$
\frac{\partial \theta}{\partial \mathrm{t}}=\frac{\partial}{\partial \mathrm{z}}\left[\mathrm{D}(\theta) \frac{\partial \theta}{\partial \mathrm{z}}-\mathrm{K}(\theta)\right]
$$

where $\mathrm{D}(\theta)$ is diffusivity defined by:

$$
\mathrm{D}(\theta)=\mathrm{K}(\theta) \frac{\partial \mathrm{h}}{\partial \theta}
$$

Equation 5, generally known as the Fokker-Planck equation (Jury 2004), is a diffusivity equation with diffusivity dependent on $\theta$. Because of this dependence, a diffusivity versus soil water content relation must be secured prior to solving equation 5 .

To define the function $\mathrm{D}(\theta)$ various expressions have been proposed. Exponential expressions proposed in 1958 by Gardner and Mayhugh (1958) have long been used, where:

$\mathrm{D}(\theta)=-\alpha \exp (\beta \theta)$

with $\alpha$ and $\beta$ constant for an assigned soil.

An exponential expression was also adopted in the theoretical studies of Scott and Hanks (1962) and Klute et al. (1965) on wetting and drying phenomena occurring in a horizontal experiment. Scott and Hanks (1962) examine a semi-infinite flow domain, with transient flow determined initially under constant water content when there occurs an unexpected variation in $\theta$ in correspondence with the initial section, at $\mathrm{x}=0$.

The following conditions are thus set for drying:

$$
\begin{gathered}
0<x<\infty \quad t>0 \\
\theta(x, t)=\theta_{0} \quad t>0 \\
\theta(x, 0)=\theta_{2}
\end{gathered}
$$

The following conditions are thus set for wetting:

$$
\begin{gathered}
0<x<\infty \quad t>0 \\
\theta(x, t)=\theta \quad t>0 \\
\theta(x, 0)=\theta_{1} \\
\text { leading to } \\
0<\theta_{2}<\theta_{0}<\theta_{1}
\end{gathered}
$$

The solution is obtained numerically with the aid of a mathematical model. For both transients, we supply graphs of the distributions of water content in the medium, using parametric curve for ratios $D_{1} / D_{0}$ or $\mathrm{D}_{0} / \mathrm{D}_{2}$ at each of the two transients in question.

For the initial section, $\mathrm{x}=0$, the infiltration velocity $i$ is expressed by the relation:

$\mathrm{i}=-\frac{\omega}{\beta} \sqrt{\frac{\alpha}{2 \mathrm{t}}}=-\frac{\omega^{*}}{\beta} \sqrt{\frac{\alpha \exp \left(\beta \theta_{0}\right)}{2 \mathrm{t}}}$

The outflow volumes $\mathrm{W}$ at time $\mathrm{t}$ are supplied by the expression:

$W(t)=-\frac{\omega^{*} A}{\beta} \sqrt{2 \alpha \exp \left(\beta \theta_{0}\right) \cdot t}$

A being the area of the transversal section of the column, $\alpha$ and $\beta$ characteristic parameters of the medium, and $\omega^{*}$ a parameter defined in drying by the ratio $D_{0} / D_{2}$ and in wetting by the ratio $D_{1} / D_{0}$.

Klute ed al. (1965) consider the same transients in a column of finite length $\mathrm{L}$, consisting of a medium of hydraulic diffusivity

$D(\theta)=D_{r} \exp (\beta) \frac{\left(\theta-\theta_{r}\right)}{\left(\theta_{s}-\theta_{r}\right)}$

$\mathrm{D}_{\mathrm{r}}$ being the diffusivity corresponding to hygroscopic water content $\theta_{\mathrm{r}}$ and $\beta$ a characteristic parameter of the porous medium.

Klute et al. (1965) also make reference to adimensional variables:

$\xi=\frac{\mathrm{x}}{\mathrm{L}}$

$\tau=\frac{D_{r}}{L^{2}}$

They then express the water volume present at time $\tau$ in the sample still susceptible of exchange with the environment by means of the relation:

$\sigma=\int_{0}^{1}\left(\theta-\theta_{\mathrm{r}}\right) /\left(\theta_{\mathrm{s}}-\theta_{\mathrm{r}}\right) \mathrm{d} \xi$

and set at the terminal sample section, $\mathrm{x}=\mathrm{L}$, the further condition of zero flow:

$\left[\frac{\partial \theta(\xi, t)}{\partial \xi}\right]_{\xi=1}=0 \quad t \geq 0$

The solution to the problem is likewise obtained numerically with the aid of the mathematical model.

For assigned values of parameter $\beta$ the distributions of water content in the medium are supplied for assigned values of $\tau$. Once again, for media with the assigned value of $\beta$, we supply the functions $\sigma(\tau)$ using the curves reported for drying and for wetting respectively in Figures 1 and 2.

Further, in the nomograph below we provide the values in time of the infiltration velocity in the initial section by means of the dimensional expression:

$$
J(\tau)=\frac{L_{i}(t)}{D_{r}\left(\theta_{s}-\theta_{r}\right)}
$$

Of particular interest is the nomograph reported in Figure 3, and that in Figure 2. It is evident that, for all the values of parameter $\beta$ considered, there may even be a $60 \%$ reduction in average water content in 
the porous medium without $J(\tau)$ being affected by the actual length $\mathrm{L}$ of the column.

In the range $1 \leq \sigma \leq 0.6$ it would thus appear appropriate to express water volumes acquired by the porous medium through more straightforward solutions relative to the semi-infinite flow domain.

\section{Materials and methods}

This study concerns the hydraulic characterisation of undisturbed terra rossa samples from Nardò in the region of Puglia (south-eastern Italy), taken from horizons Ap and B of a modal soil profile whose properties are reported in the Appendix. The samples (diameter: $80 \mathrm{~mm}$; height: $150 \mathrm{~mm}$ ) were brought to hygroscopic water content $\theta_{\mathrm{r}}$ and subjected to the wetting process from the bottom. At the end of the wetting transient, with the stabilising of condition $\theta=\theta_{1}$, the samples were brought to maximum water content $\theta_{\mathrm{s}}$, after which we proceeded to determine, under constant hydraulic head, the maximum value of hydraulic conductivity $\mathrm{K}_{\mathrm{s}}$.

Having thus impeded the flow of water at the lower base, the samples underwent the drying process. Sample drying was obtained by letting the water evaporate from the upper surface using a ventilator with a turbine of the same diameter as the sample and positioned at a height of about $12 \mathrm{~cm}$. When the ventilator is started, the drying process develops through an initial phase of constant water flow $\theta$, of little variability in relation to the properties of the sample in question (Ciollaro and Comegna, 1981). The duration $t_{c}$ of this first phase is conditioned by the possibility that, at any point of the domain, as the local value of the water content diminishes, the corresponding decrease in hydraulic conductivity $\mathrm{K}$ is compensated by the accentuation of hydraulic head gradients.

When the water content at the surface of the porous medium reaches the value corresponding to hygroscopic water content $\theta_{\mathrm{r}}$, the condition of constant flow can no longer be guaranteed. In the subsequent drying phase the transient is characterised by the parallel reduction of local gradients in the potential and in conductivity $\mathrm{K}$.

In various tests conducted on the same sample, the reduction in the initial constant water flow $\theta$ leads to a corresponding increase in duration $t_{c}$, the value $\mathrm{qt}_{\mathrm{c}}$ of the evaporated volume remaining substantially constant.

For $t>t_{c}$ the completion of drying occurs according to the law which varies little for the various values of water flow q. Yet in the same system it depends essentially on the volume of liquid still present in the same system. With the rapid exhaustion of the influence of initial conditions, there remains a curve which represents the completion of the transient with regard essentially to the characteristics of the porous medium.

In conducting our research, we examined the second phase of the transient, which is completed for $t>t_{c}$ insofar as Klute's analysis refers only to the second part of the evaporative process and may be extended to the case of vertically arranged samples, given the modest height of the dried samples. In particular, a comparison was established between wetting-drying phenomena found for the soil samples tested, according to the techniques recalled above, and those defined by using the scheme proposed by Klute. In the course of both transients, the corresponding wetting and drying functions were measured by continuously recording sample weight.

\section{Results and discussion}

In Figure 4 the two functions determined during the characterisation tests of an undisturbed red soil sample are illustrated as an exam- ple in plane $\theta(t)$. The test measurements were elaborated under the hypothesis that, for water contents close to $\theta_{\mathrm{r}}$, hydraulic diffusivity assumes the same value in both phases, namely wetting and drying.

Interpretation of the observations draws on the results of the theoretical investigations mentioned above, through calculations facilitated by the use of appropriately designed nomographs (Figures 5 and 6 ).

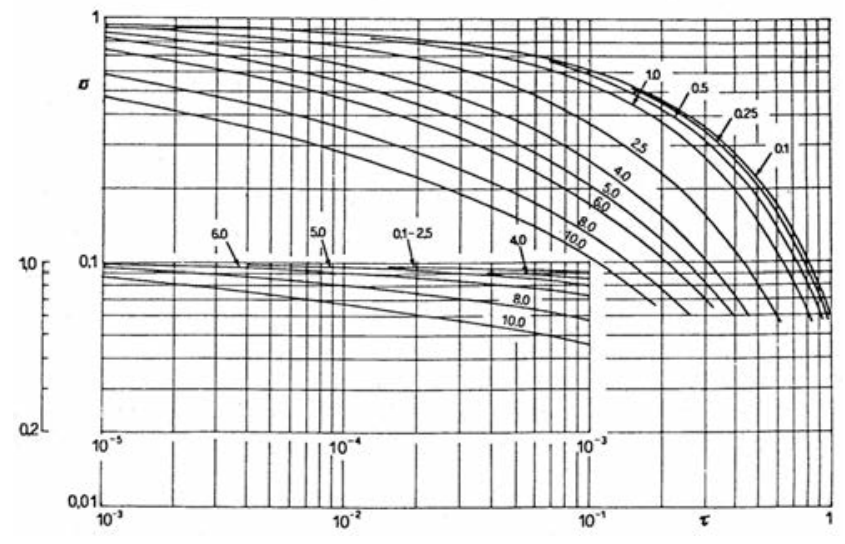

Figure 1. Values of $\sigma(\tau)$ for $\beta=$ constant, for the drying process.

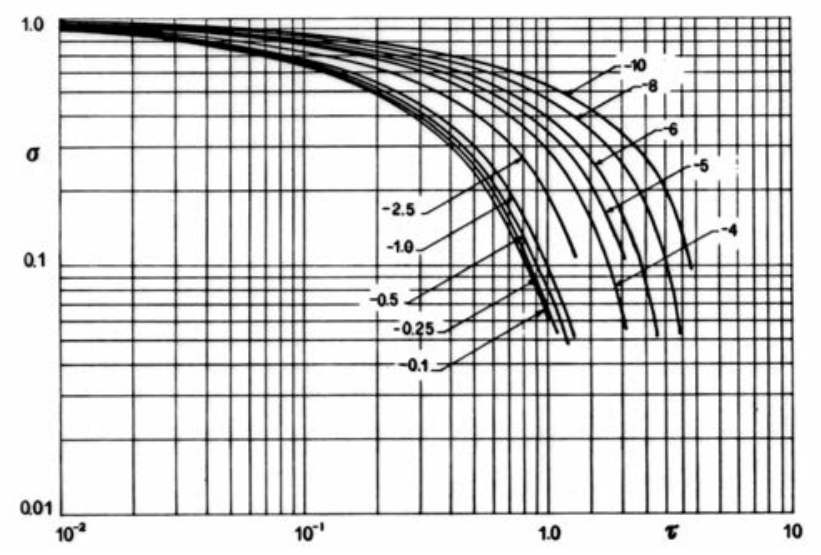

Figure 2. Values of $\sigma(\tau)$ for $\beta=$ constant, for the wetting process.

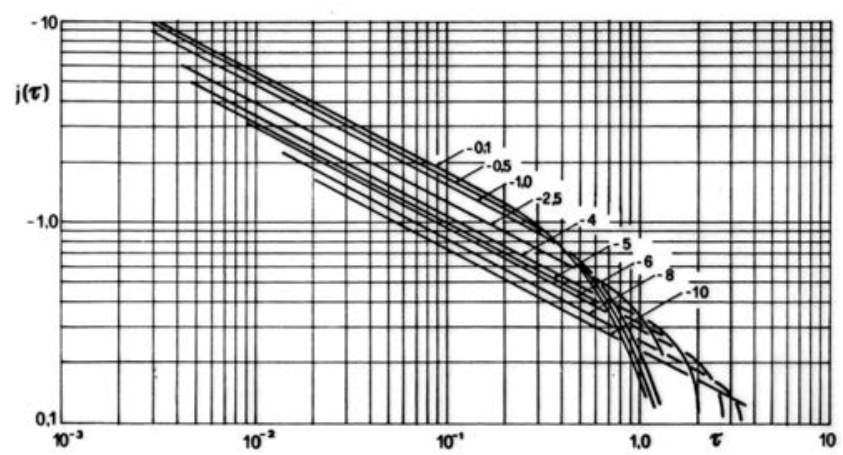

Figure 3. Values of $J(\tau)$ for $\beta=$ constant, for the wetting process. 
Use of the first (Figure 5) envisages, for longer durations $t_{c}$ of constant water flux evaporation, the acquisition of two values, $\sigma_{1}$ and $\sigma_{2}=\sigma_{1}+0.2$ and times $t_{1}, t_{2}$ and $t_{3}$ corresponding to the values $\sigma_{1}, \sigma_{2}$ and $\sigma_{3}=0.5\left(\sigma_{1}+\sigma_{2}\right)$.

On the basis of the measured value of $\sigma_{1}$ and the $\left(t_{2}-t_{1}\right) /\left(t_{3}-t_{1}\right)$ ratio using the abacus in Figure 1, by interpolation we obtain the value of parameter $\beta$. For the values of $\beta, \sigma_{1}$ and $\sigma_{2}$, once again by interpolation, we obtain the corresponding values of $\tau_{1}$ and $\tau_{2}$.

Finally, for the relation

$$
\tau=\frac{\mathrm{D}_{\mathrm{r}} \mathrm{t}}{\mathrm{L}^{2}}
$$

we arrive at determining diffusivity $D_{b}$ for $\theta=\theta_{s}$ using the expression $\mathrm{D}_{\mathrm{r}}=\left(\tau_{2}-\tau_{1}\right) \mathrm{L}^{2} /\left(\mathrm{t}_{2}-\mathrm{t}_{1}\right)$.

As regards the wetting phase, the extreme values $\theta_{\mathrm{r}}$ and $\theta_{1}$ are supplied by experience. Hence for a pair of values $\sigma$ and $\tau$, found experi-

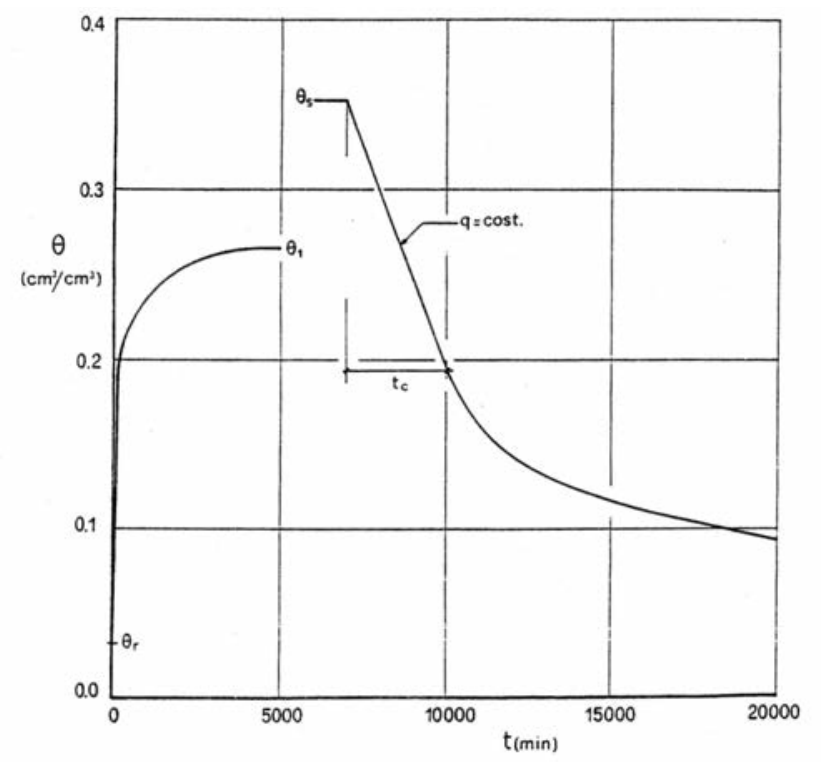

Figure 4. Laws $\theta(t)$ in wetting and subsequent drying phases.

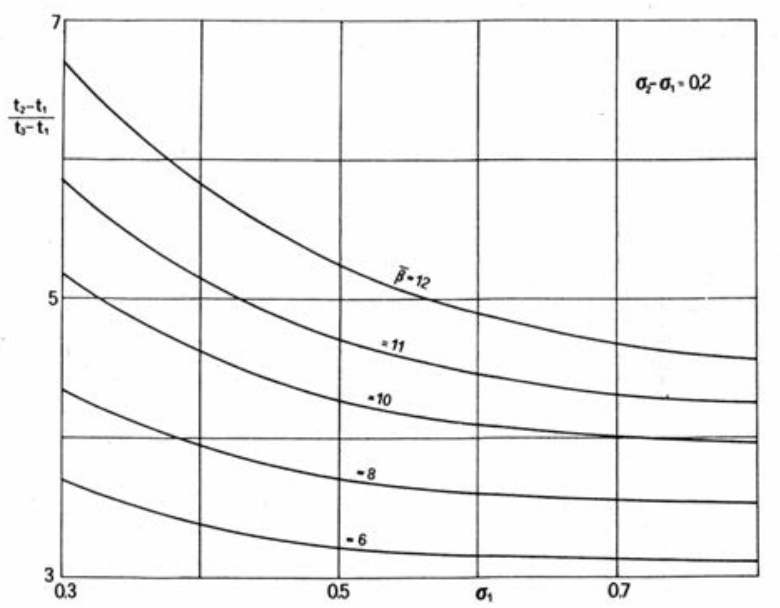

Figure 5. Values of $\frac{t_{2}-t_{1}}{t_{3}-t_{1}} v s(\sigma)$ for $\beta=$ constant. mentally, for $\sigma$ no less than 0.4 , the characteristic parameters of the medium may be determined, satisfying the equation

$\mathrm{W}=\frac{\omega^{*} \mathrm{~A}}{\beta} \sqrt{\mathrm{t}}$

in respect of $\omega^{*}$ values which are obtained by using the nomograph in Figure 6, representative of the theoretical results of Scott and Hanks.

As an example, in the ratios $\left(\theta_{\mathrm{s}}-\theta\right) /\left(\theta_{\mathrm{s}}-\theta_{\mathrm{r}}\right)$ vs D Figure 7 shows the representative curves of equation (7) obtained in characterising soil samples $\mathrm{n}^{\circ} 71,42$ and 2 .

For each sample in Table 1 we report for the applications the values assumed on the basis of soil hydraulic characterisation.

It is easy to detect that hysteresis phenomena condition the behaviour of the samples in question to a much greater extent than local variabilities which characterise natural media.

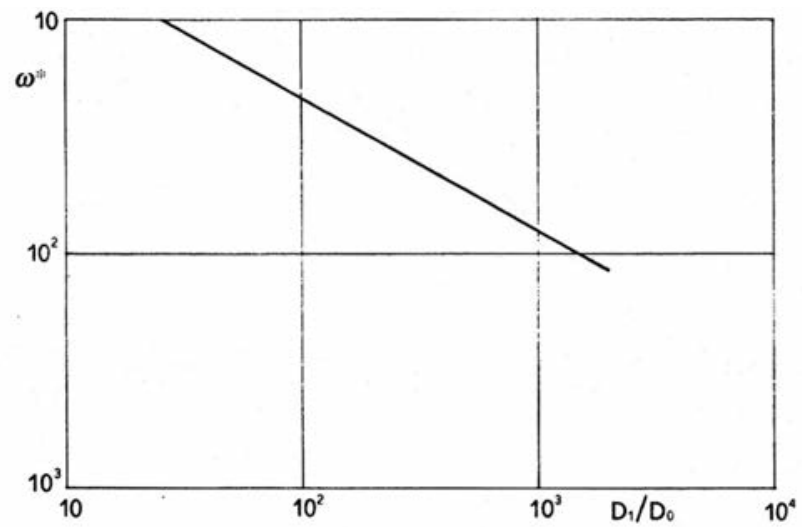

Figure 6. Values of $\omega * v s\left(D_{1} / D_{0}\right)$ in the wetting process.

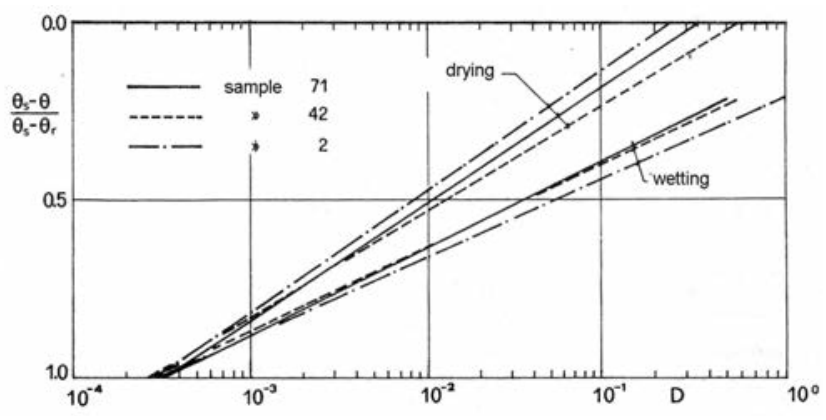

Figure 7. Values of $\left(\theta_{\mathrm{s}}-\theta\right) /\left(\theta_{\mathrm{s}}-\theta_{\mathrm{r}}\right) v s D$. 
Table 1. Basic soil hydraulic properties.

\begin{tabular}{|c|c|c|c|c|c|c|c|}
\hline $\begin{array}{l}\text { Sample } \\
\text { No. }\end{array}$ & $\begin{array}{c}\theta_{\mathrm{r}} \\
\mathrm{cm}^{3} / \mathrm{cm}^{3}\end{array}$ & $\begin{array}{c}\theta_{1} \\
\mathrm{~cm}^{3} / \mathrm{cm}^{3}\end{array}$ & $\begin{array}{c}\theta_{\mathrm{s}} \\
\mathrm{cm}^{3} / \mathrm{cm}^{3}\end{array}$ & $\begin{array}{c}D_{\mathrm{r}} \\
\mathrm{cm}^{2} / \min \end{array}$ & $\begin{array}{c}\mathrm{D}_{1} \\
\mathrm{~cm}^{2} / \mathrm{min}\end{array}$ & $\begin{array}{c}D_{\mathrm{s}} \\
\mathrm{cm}^{2} / \mathrm{min}\end{array}$ & $\begin{array}{c}\mathrm{K}_{\mathrm{s}} \\
\mathrm{cm} / \mathrm{min}\end{array}$ \\
\hline 71 & 0.031 & 0.264 & 0.351 & 0.00030 & 0.45 & 0.33 & 0.0253 \\
\hline 42 & 0.036 & 0.280 & 0.329 & 0.00025 & 0.50 & 0.57 & 0.0852 \\
\hline 2 & 0.037 & 0.25 & 0.345 & 0.00027 & 1.00 & 0.23 & 0.0469 \\
\hline
\end{tabular}

\section{Conclusions}

The test method proposed requires that only the sample's mean water content be recorded by weighing. Experimental findings highlighted the validity of wetting and subsequent drying test in hydraulic characterization of soil samples.

Interpretation of the results, developed in light of theoretical data, allows straightforward definition of both primary wetting and drying curves of hydraulic diffusivity of the medium. It is easy to detect that hysteresis conditions the behaviour of the samples in question to a much greater extent than local variabilities which characterise natural media. Our results show that it may be useful to extend observations to other soil types. The proposed method may be considered an effective investigation tool when hydraulic characterisation of different pedological units is carried out on a statistical basis.

\section{Appendix}

Sample ${ }^{\circ}$ 71) Undisturbed, sampled at Nardò (LE) from horizon Ap, between the depths of 10 and $25 \mathrm{~cm}$; classified as sandy (SISS): $81 \%$ sand; $6 \%$ silt; $13 \%$ clay; height $\mathrm{L}=11 \mathrm{~cm}$; bulk density $\mathrm{r}=1.59 \mathrm{~g} / \mathrm{cm}^{3}$.

Sample $n^{\circ} 42$ ) Undisturbed, sampled at Nardò from horizon $B$, between the depths of 25 and $40 \mathrm{~cm}$; classified as sandy-clayey (SISS): $76 \%$ sand; $6 \%$ silt; $18 \%$ clay; height $\mathrm{L}=13 \mathrm{~cm}$; bulk density $\mathrm{r}=1.57 / \mathrm{cm}^{3}$.

Sample $n^{\circ}$ 2) Undisturbed, sampled at Nardò from horizon $B$, between the depths of 50 and $65 \mathrm{~cm}$; classified as sandy-clayey (SISS): $76 \%$ sand; $6 \%$ silt; $18 \%$ clay; height $\mathrm{L}=12.5 \mathrm{~cm}$; bulk density $\mathrm{r}=1.52 \mathrm{~cm}^{3}$.

\section{References}

Childs E.C., Collis-George C. 1950. The permeability of porous materials. Proc. R. Soc. London Ser.(A). 201: 392-405.

Ciollaro G., Comegna V. 1981. La caratterizzazione idraulica del suolo nell'intervento irriguo. Pubblicazione dell'Istituto di Idraulica Agraria di Napoli. Università di Napoli.

Ciollaro G., Romano N. 1995. Spatial variability of the hydraulic properties of a volcanic soil. Geoderma. 65: 263-282.

Coppola A., Gerke H.H., Comegna A., Basile A., Comegna V. 2012. Dualpermeability model for flow in shrinking soil with dominant hori- zontal deformation. Water Resour. Res. 48: W08527, doi:10.1029/ 2011WR011376.

Doering E.J. 1965. Soil-water diffusivity by the one-step method. Soil Sci. 99:322-326.

Eching S.O., Hopmans J.W., Wendroth 0. 1994. Unsaturated hydraulic conductivity from transient multistep outflow and soil water pressure data. Soil Sci. Soc. Am. J. 58: 687-695.

Gardner W.R., 1956. Calculation of capillary conductivity from pressure outflow data. Soil Sci. Soc. Am. Proc. 20: 317-320.

Gardner W.R., Mayhugh M.S. 1958. Solutions and tests of the diffusion equation for the movement of water in soil: Soil Sci. Soc. Am. Proc. 22: 197-201.

Gardner W.R. 1962. Note on the separation and solution of diffusion type equations. Soil Sci. Soc. Am. Proc. 26: 404.

Gupta S.C., Farrel D.A., Larson W.E. 1974. Determining effective soil water diffusivities from one-step outflow experiments. Soil Sci. Soc. Am. J. 38: 710-716.

Jury W.A., Horton R. 2004. Soil Physics, John Wiley and Sons, Inc.

Klute A., Whisler F.D., Scott E.J. 1965. Numerical solution of the nonlinear diffusion equation for water flow in a horizontal soil column of finite length. Soil Sci. Soc. Am. Proc. 4: 353-358.

Kool J.B., Parker J.C., van Genuchten M.Th. 1987. Parameter estimation for unsaturated flow and transport models-A review. J. Hydrol. 91: 255-293.

Kunze R.J., Kirkham D. 1962. Simplified accounting for membrane impedance in capillary conductivity determinations. Soil Sci. Soc. Am. Proc. 26: 421-426.

Londra P.A., Valiantzas, J.D. 2011. Soil water diffusivity determination using a new two-point outflow method. Soil Sci. Soc. Am. J., 75: $1343-1346$.

Scott E.J., Hanks R.J. 1962 Solution of the one dimensional diffusion equation for exponential and linear diffusivity functions by power series applied to moisture flow in soils. Soil Sci. 94: 314-322.

Parker J.C., Kool J.B., van Genuchten M.Th. 1985. Determining soil hydraulic properties from one-step outflow experiments by parameter estimation: II. Experimental studies. Soil Sci. Soc. Am. J. 49: 1354-1359.

Passioura J.B. 1976. Determining soil water diffusivities from one step outflow experiments. Aust. J. Soil Res. 15:1-8. doi:10.1071/ SR9770001.

Rijtema P.E. 1959. Calculation of capillary conductivity from pressure plate outflow data with non-negligible membrane impedance. Netherlands J. Agr, Sci. 7:209-215.

Valiantzas J.D. 1990. Analysis of outflow experiments subject to significant plate impedance. Water Resour. Res. 26:2921-2929. doi:10.1029/ WR026i012p02921. 